\title{
Leadless pacemakers in critically ill patients requiring prolonged cardiac pacing; a multicenter international study.
}

Nicolas Clementy ${ }^{1}$, Roxane Coelho ${ }^{1}$, Christian Veltmann ${ }^{2}$, Eloi Marijon ${ }^{3}$, Jose Maria Tolosana ${ }^{4}$, Vincent Galand ${ }^{5}$, Sylvain Ploux ${ }^{6}$, Romain Eschalier ${ }^{7}$, Edouard Simeon ${ }^{8}$, Hermann Blessberger ${ }^{9}$, Johanna Müller-Leisse ${ }^{2}$, Margarida Pujol-Lopez ${ }^{10}$, Raphael Martins $^{5}$, Philippe Ritter ${ }^{11}$, Clemens Steinwender ${ }^{12}$, and Dominique Babuty ${ }^{13}$

${ }^{1}$ CHU Trousseau

${ }^{2}$ Hannover Medical School

${ }^{3}$ Hopital Europeen Georges Pompidou

${ }^{4}$ Hospital Clinic, Universitat de Barcelona;

${ }^{5}$ CHU Rennes

${ }^{6} \mathrm{CHU}$ Bordeaux, Hopital Haut Leveque

${ }^{7}$ Clermont Université

${ }^{8}$ Institut Mutualiste Montsouris

${ }^{9}$ Linz General Hospital

${ }^{10}$ Hospital Clinic de Barcelona

${ }^{11} \mathrm{CHU}$ de Bordeaux Hôpital Cardiologique

${ }^{12}$ Johannes Kepler Universitat Linz

${ }^{13}$ François Rabelais University

March 2, 2021

\begin{abstract}
Background: Temporary transvenous pacing in critically ill patients requiring prolonged cardiac pacing is associated with a high risk of complications. We sought to evaluate the safety and efficacy of self-contained intracardiac leadless pacemaker (LPM) implantation in this population. Methods and Results: Consecutive patients implanted with a Micra LPM during the hospitalization in an intensive care unit were retrospectively included. Inclusion criteria were: [?]1 supracaval central venous, or a ventilation tube, or intravenous antibiotic therapy for ongoing sepsis or bacteremia. Patients with a history of previous implantation of a pacemaker were excluded. Out of 1,016 patients implanted with an LPM, 99 met the inclusion criteria. Mean age was 75 years and Charlson comorbidity index 7. LPM implantation was successfully performed in $98 \%$ of cases, with a peri-operative complication rate of $5 \%$, mainly cardiac injuries. In-hospital mortality rate was $6 \%$. No late ( $>30$ days) devicerelated complication occurred, especially no infection. Conclusions: LPM appears as an acceptable alternative to conventional temporary transvenous pacing in selected critically ill patients requiring prolonged cardiac pacing, especially regarding the risk of infection.
\end{abstract}

Title: Leadless pacemakers in critically ill patients requiring prolonged cardiac pacing; a multicenter international study.

Authors: Nicolas CLEMENTY, ${ }^{1}$ Roxane COELHO, ${ }^{1}$ Christian VELTMANN ${ }^{3}$ Eloi MARIJON,${ }^{4}$ José TOLOSANA, ${ }^{5}$ Vincent GALAND,${ }^{6}$ Sylvain PLOUX, ${ }^{7}$ Romain ESCHALIER, ${ }^{8}$ Edouard SIMEON,${ }^{9}$ 
Hermann BLESSBERGER, ${ }^{2}$ Johanna MUELLER-LEISSE, ${ }^{3}$ Margarida PUJOL-LOPEZ, ${ }^{5}$ Raphaël MARTINS, ${ }^{6}$ Philippe RITTER, ${ }^{7}$ Clemens STEINWENDER,${ }^{2}$ and Dominique BABUTY. ${ }^{1}$

Affiliations: ${ }^{1}$ Department of Cardiology, University Hospital of Tours, Tours, France;

2 Department of Cardiology, Kepler University Hospital Linz, Linz, Austria;

${ }^{3}$ Department of Cardiology and Angiology, Hannover Medical School, Hannover, Germany;

${ }^{4}$ Department of Cardiology, European Georges Pompidou Hospital, Paris, France;

${ }^{5}$ Hospital Clinic, University of Barcelona, Barcelona, Spain;

${ }^{6}$ Department of Cardiology, University Hospital Pontchaillou, Rennes, France;

${ }^{7}$ Cardio-Thoracic Unit, Bordeaux University Hospital (CHU), Pessac, France;

${ }^{8}$ Department of Cardiology, University Hospital Clermont-Ferrand, Clermont-Ferrand, France;

${ }^{9}$ Department of Electrophysiology, Private Hospital Institute Mutualiste Montsouris, Paris, France.

Short Title: Leadless pacemakers in ICU.

Word Count: 3,457

Corresponding Author: Nicolas CLEMENTY

Address: Service de Cardiologie, Hôpital Trousseau, 37044 Tours, France

Telephone: $+33-247474687$

Fax: $+33-247475919$

E-mail: nclementy@yahoo.fr

ORCID: 0000-0002-7415-0787

\section{DECLARATIONS}

\section{Acknowledgements}

None.

Funding

None.

\section{Conflicts of interest/Competing interests (include appropriate disclosures)}

Nicolas CLEMENTY received honoraria for lectures/consulting from Medtronic; Christian VELTMANN received honoraria for lectures/consulting from Medtronic; Johanna MUELLER-LEISSE received honoraria for lectures/consulting from Medtronic; Clemens STEINWENDER received honoraria for lectures/consulting from Medtronic. All other authors report no disclosure.

\section{Authors' contributions}

Nicolas CLEMENTY, Roxane COELHO, and Dominique BABUTY contributed substantially to the conception and design of the study, the acquisition of data, the analysis and interpretation of the data, drafted the manuscript, and provided critical revision of the article.

Christian VELTMANN, Eloi MARIJON, José TOLOSANA, Vincent GALAND, Sylvain PLOUX, Romain ESCHALIER, Edouard SIMEON, Hermann BLESSBERGER, Johanna MUELLER-LEISSE, Margarida PUJOL-LOPEZ, Raphaël MARTINS, and Clemens STEINWENDER contributed substantially to the acquisition of data, and provided critical revision of the article. 
All authors provided final approval of the version submitted for publication.

\section{ABSTRACT}

Background: Temporary transvenous pacing in critically ill patients requiring prolonged cardiac pacing is associated with a high risk of complications. We sought to evaluate the safety and efficacy of self-contained intracardiac leadless pacemaker (LPM) implantation in this population.

Methods and Results: Consecutive patients implanted with a Micra LPM during the hospitalization in an intensive care unit were retrospectively included. Inclusion criteria were: [?]1 supracaval central venous, or a ventilation tube, or intravenous antibiotic therapy for ongoing sepsis or bacteremia. Patients with a history of previous implantation of a pacemaker were excluded. Out of 1,016 patients implanted with an LPM, 99 met the inclusion criteria. Mean age was 75 years and Charlson comorbidity index 7. LPM implantation was successfully performed in $98 \%$ of cases, with a peri-operative complication rate of $5 \%$, mainly cardiac injuries. In-hospital mortality rate was $6 \%$. No late ( $>30$ days) device-related complication occurred, especially no infection.

Conclusions: LPM appears as an acceptable alternative to conventional temporary transvenous pacing in selected critically ill patients requiring prolonged cardiac pacing, especially regarding the risk of infection.

\section{KEY WORDS}

Leadless; pacemaker; intensive care; temporary pacing.

\section{Unstructured Abstract}

Temporary transvenous pacing in critically ill patients requiring prolonged cardiac pacing is associated with a high risk of complications. Leadless pacemaker implantation in patients hospitalized in intensive care unit is feasible in almost $100 \%$ of cases and associated with a $5 \%$ procedure-related complications rate. It appears as an interesting alternative to conventional temporary transvenous pacing in selected critically ill patients requiring prolonged cardiac pacing, to reduce the risk of both acute and late complications, especially infections and pacing failures.

\section{What's New?}

Leadless pacemakers can be safely implanted in ICU patients requiring prolonged pacing, with a $<5 \%$ complication rate, and no infection.

A permanent leadless pacemaker can be proposed as an alternative to temporary transvenous pacing to critically-ill patients with persistent symptomatic bradycardia episodes.

\section{ABBREVIATION LIST}

ICU: intensive care unit

IQR: interquartile range

LPM: leadless pacemaker

PPM: permanent cardiac pacemaker

SD: standard deviation

TTP: transvenous temporary pacing

\section{INTRODUCTION}

Critically ill patients may present a permanent, or at least prolonged, indication of cardiac pacing, either because they are hospitalized following severe bradycardia, or because they incidentally develop repetitive or persistent bradycardia episodes in addition to a primary severe medical condition [1]. These patients 
may initially require transvenous temporary pacing (TTP), and if bradycardia persists, eventually the implantation of a permanent cardiac pacemaker (PPM). Conventional transvenous PPM implantation can be challenging in patients with multiple central venous lines. It is also associated with an increased risk of severe complications, such as pneumo/haemothorax, haematoma, cardiac injury, lead dislodgement, and of course infection (local pocket and/or lead endocarditis) which often drives the prognosis in this population $[2]$.

Leadless pacemakers (LPMs) have recently emerged as a useful tool in patients requiring a permanent single-chamber pacing device [3,4]. They have been shown to be associated with a low rate of complications, especially a virtually zero risk of infection, even in patients with a previous device infection $[5,6]$. Moreover, as their implantation is performed through a transvenous femoral approach, they are first-choice devices in patients with unsuitable supracaval venous access.

We sought to evaluate the safety and efficacy of LPM implantation in the high-risk population of patients hospitalized in intensive care unit (ICU) who require prolonged cardiac pacing.

\section{METHODS}

\section{Study population}

Patients implanted with the Micra Transcatheter Pacing System (Medtronic, Minneapolis, MN, USA), from 9 major tertiary European LPM implanting centers, between September 2015 and August 2019 (4 years), and followed in the Micra Transcatheter Pacing System Post-Approval Registry (NCT02536118) were retrospectively screened.

Inclusion criteria were: patients with an indication of prolonged cardiac pacing, who underwent an LPM implantation during the hospitalization in an ICU; and the presence of at least one of the following: [?]1 supracaval central venous line (including hemodialysis catheter), or a ventilation tube, or intravenous antibiotic therapy for ongoing sepsis or bacteremia. Exclusion criteria were: previous implantation of a pacemaker. This study was conducted in accordance with the amended Declaration of Helsinki, and written informed consent was obtained from all patients or their substitute decision-makers.

Data on clinical characteristics of patients, especially comorbidities, LPM implantation procedure, duration of hospitalization were collected. Patients had regular cardiovascular consultation including LPM interrogation of electrical parameters ([?]1 per year).

\section{Statistical analyses}

The analyses were performed on JMP 9.0 software (SAS Inc., Cary, NC, USA). Continuous data were presented as median and standard deviation (SD) or interquartile range (IQR). Categorical data were reported as frequencies and percentages. Comparisons used the $\chi^{2}$ or Fisher's exact test for categorical variables and Student's t test or Mann-Whitney-Wilcoxon test, when appropriate, for continuous variables. Survival curves were calculated using the Kaplan-Meier method. A two-tailed p-value $<0.05$ was considered statistically significant.

\section{RESULTS}

\section{Population}

A total of 1,016 consecutive patients implanted with a Micra TPS LPM during the inclusion period were screened, of whom $99(9.7 \%)$ met the inclusion criteria. Clinical characteristics of patients are reported inTable 1 .

The primary diagnosis for admission in ICU was cardiovascular in 86 patients (87\%): extreme bradycardia $(n=30)$ or cardiac arrest $(n=2)$, ventricular arrhythmias $(n=2)$, post-operative following transcatheter aortic valve replacement $(n=26)$ or open-chest cardiac surgery $(n=8)$, cardiogenic shock $(n=8)$ or heart failure $(\mathrm{n}=3)$, and endocarditis $(\mathrm{n}=7)$. Other main diagnoses were: septic shock $(\mathrm{n}=2)$ or sepsis $(\mathrm{n}=1)$ (excluding 
endocarditis), acute respiratory distress syndrome $(\mathrm{n}=3)$, post-operative following abdominal surgery $(\mathrm{n}=3)$, ketoacidosis $(n=1)$, hypovolemic shock $(n=1)$, hepatic encephalopathy $(n=1)$, and severe burn $(n=1)$.

\section{Implantation}

LPM implantation was performed a median of 8 days following admission in ICU (range 1-180, IQR 10 days) (Table 2 ). Indication was high degree atrioventricular block in 86 patients, and sick sinus syndrome in 13. Only $52 \%$ of patients had a concomitant atrial arrhythmia.

LPM implantation was successfully performed in all but 2 cases (98\%): no adequate acute pacing threshold ([?]2.0 volts) could be obtained after up to 15 different positionings in 1 patient; the tricuspid valve could not be crossed in 1 patient with a history of heart transplantation. Approach was through the right femoral vein in 95 patients, left femoral vein in 4 . A total of $71 \%$ of patients underwent a single positioning before reaching a final position (91\% interventricular septum, $9 \%$ right ventricular apex), the total of positionings being [?]2 in $88 \%$ of cases. Median pacing threshold at implantation was as low as 0.38 volt, with 0.24 millisecond of pacing spike duration (range $0.13-1.63$, IQR 0.25 volt).

A total of 4 procedure-related cardiac injuries occurred: 3 patients with a tamponade requiring percutaneous pericardial drainage, and 1 with a well-tolerated pericardial effusion (Table 3 ). One patient died, an 82year-old woman hospitalized following a prolonged cardiac arrest due to complete atrioventricular block: a cardiac tamponade after the procedure was successfully drained, however she developed rapid lethal multisystem organ failure. Patients with cardiac injury were significantly more likely to have diabetes $(75 \%$ versus $26 \%, \mathrm{p}=0.04)$, liver failure $(75 \%$ versus $5 \%, \mathrm{p}=0.001)$ and normal coronary arteries $(100 \%$ versus $42 \%, \mathrm{p}=0.0002)$.

No complication related to the vascular access (groin hematoma, fistula, etc) occurred.

\section{Follow-up}

The median follow-up period was 19 months (IQR 40 months).

Among the 97 patients successfully implanted with an LPM, 6 (6.1\%) died during hospitalization after a median of 17 days (range 1-37, IQR 22 days): 1 from a procedure-related tamponade, and 5 from ongoing septic shock unrelated to the implantation procedure. One patient had a high rise of the pacing threshold necessitating the implantation of a conventional pacemaker 4 days following LPM implantation (Table 3 ). In-hospital and 30-day mortality rates were 6 and 7\%, respectively (Figure 1 ).

Ninety-one patients were discharged after a median of 5 days following LPM implantation (range 1-38, IQR 6 days). All discharged patients had excellent electrical performances at first follow-up visit with a median of 0.5 volt of pacing threshold at 0.24 millisecond (range 0.13-1.13, IQR 0.12 volt). Median percentage of ventricular pacing was $28 \%, 46 \%$ of patients being considered having permanent pacing ([?]90\% of the time), and $54 \%$ rare ventricular pacing ([?]10\% of the time). Nine patients died during follow-up after discharge (10\%) a median of 191 days (range 22-1,219, IQR 505 days) following discharge, none being related to the implantation procedure or the pacing device: refractory cardiogenic shock in 5 patients $(22,90,191,240$, and 637 days post-implantation), traumatic brain injury in 1 (55 days), pulmonary insufficiency in 1 (157 days), suicide in 1 (518 days), and pulmonary infection in 1 (1,219 days). One-year mortality rate was $16 \%$ (Figure 1 ).

No late ( $>30$ days) device-related complication occurred, especially no infection. No pacemaker syndrome or any upgrade to a dual-chamber or biventricular device was reported.

\section{DISCUSSION}

Our main results are the following: 1) permanent LPM implantation in patients hospitalized in intensive care unit is feasible in $98 \%$ of cases; 2 ) procedure-related complications are $<5 \%$ in this very high-risk population, with a virtually null risk of infection. 
Pacemaker implantation in ICU patients is associated with several challenging issues. Venous access is often limited due to the presence of central venous lines for perfusion or hemodialysis or hemofiltration $[7,8]$. In our series, 3 patients had no suitable supracaval venous access for conventional pacemaker implantation. The alternative is cardiac surgery with an epicardial approach, which appears highly invasive in this critically ill population. The infectious risk is also a major issue, amplified by multiple central venous lines, mechanical ventilation, and ongoing sepsis or bacteremia [9-11]. More than half of our patients were actually under intravenous antibiotherapy.

When atropine bolus or isoproterenol infusion are not sustainable options, implantation of a temporary pacemaker is usually performed, before considering PPM [12]. TTP is associated with a high rate of complications [13]. In the US national database, pneumothorax, pericardial tamponade and non-pericardial bleeding were found in $0.6,0.9$ and $2.4 \%$ of patients, respectively [14]. In a more recent review of the literature, the total rate of complications related to TTP ranged from $23 \%$ (in the 2010 s decade, $5.7 \%$ considered serious complications) to $43 \%$ (in the $1980 \mathrm{~s}, 16.5 \%$ serious), including $2 \%$ of complicated access, $1.6 \%$ of cardiac perforation, near $5 \%$ of infections, and more than $20 \%$ of device complications [2]. TTP also prolongs hospitalization with a mean of 11 days.

Patients hospitalized in ICU and requiring prolonged cardiac pacing are frail and severe. Bradycardia $<40$ beats-per-minute adds 4 points to the APACHE II and 11 points to the SAPS II scores. In our cohort, median Charlson comorbidity index was as high as 7 and in-hospital mortality as low as $6 \%$, as compared with a median score of 2 and an in-hospital mortality rate of $18 \%$ in a study by Christensen and colleagues, although these 2 ICU populations cannot be directly compared [15-17].

In that respect, LPM implantation is appealing. The Micra LPM is a small 0.8 cubic centimeter device, weighing barely 2 grams, implanted through a femoral approach on the right ventricular septum (Figure 2 ). The femoral approach solves the potential issues regarding supracaval venous access, and the risk of device-related infection is virtually nil, even in high-risk populations $[6,18]$. In our series, $60 \%$ of patients had ongoing sepsis or documented bacteremia, of whom $92 \%$ were under antibiotherapy, and no device infection occurred. Moreover, the risk of dislodgement or pacing issue is extremely rare $(<1 \%)[19]$, to be compared with a risk of $\sim 20 \%$ with TTP [2]. The main issue with LPM is the risk of acute cardiac injury, $4 \%$ in our series, as compared with a less than $1 \%$ reported rate in the literature [18]. This can be easily explained by the frailty and multiple comorbidities of the ICU population. This risk could be further decreased by positioning the device on the right ventricular septum rather than at the apex, and with regular appropriate training [19].

The alternative to implant an active fixation lead connected to an external re-sterilized pulse generator has also been shown to be associated with a very low risk of complications, while being a removable system [20]. However, this technique requires suitable supracaval access and could not have been offered in up to $3 \%$ of our patients.

\section{Limitations}

Although this multicenter European registry included most of the main LPM implanting centers, it may not reflect the general population requiring permanent pacing in ICU. LPM, which is not to be removed, is a permanent device, whereas TTP is only followed by PPM implantation in roughly 2 thirds of cases [2]. This technique also raises the question about the overall procedure cost. Finally, all LPMs implanted in this study were single-chamber device, even in sinus rhythm patients with complete atrioventricular block. The next generation of LPM with atrioventricular synchronization should solve this potential issue [21].

\section{Conclusions}

LPM implantation in ICU patients requiring prolonged cardiac pacing is feasible and was associated with a limited number of serious complications in this very high-risk population. It appears as an acceptable alternative to TTP in selected patients, especially regarding the risk of infection.

\section{REFERENCES}


1. Reinelt P, Karth GD, Geppert A, Heinz G. Incidence and type of cardiac arrhythmias in critically ill patients: a single center experience in a medical-cardiological ICU. Intensive Care Med. 2001;27:1466-73.

2. Tjong FVY, de Ruijter UW, Beurskens NEG, Knops RE. A comprehensive scoping review on transvenous temporary pacing therapy. Neth Heart J. 2019;27:462-73.

3. Reddy VY, Exner DV, Cantillon DJ, et al. Percutaneous Implantation of an Entirely Intracardiac Leadless Pacemaker. N Engl J Med. 2015;373:1125-35.

4. Reynolds D, Duray GZ, Omar R, et al. A Leadless Intracardiac Transcatheter Pacing System. N Engl J Med. 2016;374:533-41.

5. Kypta A, Blessberger H, Kammler J, et al. Leadless Cardiac Pacemaker Implantation After Lead Extraction in Patients With Severe Device Infection. J Cardiovasc Electrophysiol. 2016;27:1067-71.

6. El-Chami MF, Johansen JB, Zaidi A, et al. Leadless pacemaker implant in patients with pre-existing infections: Results from the Micra postapproval registry. J Cardiovasc Electrophysiol. 2019;30:569-74.

7. Bhadauria D, Chellappan A, Gurjar M, Kaul A, Sharma RK, Prasad N. The "dilemma of double lifelines": central venous catheter co-existence with transvenous cardiac pacemaker. J Vasc Access. 2017;18:e3-5.

8. Roig IL, Darouiche RO, Musher DM, Trautner BW. Device-related infective endocarditis, with special consideration of implanted intravascular and cardiac devices in a predominantly male population. Scand J Infect Dis. 2012;44:753-60.

9. Marik PE, Flemmer M, Harrison W. The risk of catheter-related bloodstream infection with femoral venous catheters as compared to subclavian and internal jugular venous catheters: a systematic review of the literature and meta-analysis. Crit Care Med. 2012;40:2479-85.

10. Fayssoil A, Lazarus A, Wahbi K, et al. Cardiac implantable electronic devices in tracheotomized muscular dystrophy patients: Safety and risks. Int J Cardiol. 2016;222:975-7.

11. Polyzos KA, Konstantelias AA, Falagas ME. Risk factors for cardiac implantable electronic device infection: a systematic review and meta-analysis. Europace. 2015;1:767-77.

12. Sodeck GH, Domanovits H, Meron G, et al. Compromising bradycardia: management in the emergency department. Resuscitation. 2007;73:96-102.

13. Ng ACC, Lau JK, Chow V, Adikari D, Brieger D, Kritharides L. Outcomes of 4838 patients requiring temporary transvenous cardiac pacing: A statewide cohort study. Int J Cardiol. 2018;271:98-104.

14. Metkus TS, Schulman SP, Marine JE, Eid SM. Complications and Outcomes of Temporary Transvenous Pacing: An Analysis of > 360,000 Patients From the National Inpatient Sample. Chest. 2019;155:749-57.

15. Charlson ME, Pompei P, Ales KL, MacKenzie CR. A new method of classifying prognostic comorbidity in longitudinal studies: development and validation. J Chronic Dis. 1987;40:373-83.

16. Stavem K, Hoel H, Skjaker SA, Haagensen R. Charlson comorbidity index derived from chart review or administrative data: agreement and prediction of mortality in intensive care patients. Clin Epidemiol. 2017;9:311-320.

17. Christensen S, Johansen MB, Christiansen CF, Jensen R, Lemeshow S. Comparison of Charlson comorbidity index with SAPS and APACHE scores for prediction of mortality following intensive care. Clin Epidemiol. 2011;3:203-11.

18. El-Chami MF, Clementy N, Garweg C, et al. Leadless Pacemaker Implantation in Hemodialysis Patients: Experience With the Micra Transcatheter Pacemaker. JACC Clin Electrophysiol. 2019;5:162-70.

19. Roberts PR, Clementy N, Al Samadi F, et al. A leadless pacemaker in the real-world setting: The Micra Transcatheter Pacing System Post-Approval Registry. Heart Rhythm. 2017;14:1375-9. 
20. Zei PC, Eckart RE, Epstein LM. Modified temporary cardiac pacing using transvenous active fixation leads and external re-sterilized pulse generators. J Am Coll Cardiol. 2006;47:1487-9.

21. Chinitz L, Ritter P, Khelae SK, et al. Accelerometer-based atrioventricular synchronous pacing with a ventricular leadless pacemaker: Results from the Micra atrioventricular feasibility studies. Heart Rhythm. 2018;15:1363-71.

\section{FIGURE LEGENDS}

Figure 1. Kaplan-Meier survival curve from all-cause death following leadless pacemaker implantation. Central panel : zoom in perioperative period ([?]30 days post- implantation).

Figure 2. Right upper panel : the Micra transcatheter pacing system is a small 26 millimeters long pacing device (0.8 cubic centimeters, 2 grams), with nitinol tines allowing passive fixation within myocardium. Right lower panel : usual final positioning of leadless pacemakers on the right ventricular low septum, through a femoral venous approach. Left panel : chest X-Ray (film negative) of a patient implanted with a leadless pacemaker (LPM), with right and left jugular central lines (RJVL and LJVL, respectively), and an orotracheal ventilation tube (VT).

\section{TABLES}

Table 1. Patients' characteristics at admission.

\begin{tabular}{ll}
\hline & ALL (N=99) \\
\hline Age (years) & $75 \pm 13$ \\
Male gender (\%) & $51(52)$ \\
Body mass index $\left(\mathrm{kg} . \mathrm{m}^{-2}\right)$ & $27 \pm 6$ \\
Heart failure (\%) & $32(32)$ \\
Ischemic heart disease (\%) & $55(56)$ \\
Valvular heart disease (\%) & $45(45)$ \\
Left ventricular ejection fraction (\%) & $55 \pm 11$ \\
Atrial fibrillation (\%) & $52(53)$ \\
Chronic renal failure (\%) & $59(60)$ \\
eGFR (mL.min 1 .1.73m & -2 \\
Chronic liver failure (\%) & $56 \pm 30$ \\
Chronic pulmonary disease (\%) & $8(8)$ \\
Diabetes mellitus (\%) & $26(26)$ \\
Cancer (\%) & $28(28)$ \\
Antiplatelet treatment x1 / x2 (\%) & $18(18)$ \\
Anticoagulation DOAC / VKA (\%) Charlson Comorbidity Index & $27(27) / 23(23)$ \\
\hline
\end{tabular}

eGFR, estimated glomerular filtration rate (MDRD); DOAC, direct oral anticoagulant; VKA, vitamin-K antagonist.

Table 2 . Patients' characteristics at pacemaker implantation.

\begin{tabular}{ll}
\hline & ALL (N=99) \\
\hline Pacing indication SSS / AVB (\%) & $13(13) / 86(87)$ \\
Central venous line 0 / 1 / 2 (\%) & $41(41) / 53(54) / 5(5)$ \\
Femoral right / left (\%) & $3(3) / 12(12)$ \\
Subclavian right / left (\%) & $6(6) / 1(1)$ \\
Jugular right / left (\%) & $32(32) / 6(6)$ \\
PICC line (\%) & $3(3)$
\end{tabular}




\begin{tabular}{ll}
\hline & ALL (N=99) \\
\hline Hemodialysis or hemofiltration (\%) No suitable supracaval venous access (\%) & $8(8) 3(3)$ \\
Mechanical ventilation (\%) & $16(16)$ \\
Orotracheal (\%) & $10(10)$ \\
Tracheotomy (\%) & $6(6)$ \\
Deep sedation (\%) & $11(11)$ \\
Bacteremia / Sepsis / Septic shock (\%) & $40(40) / 17(17) / 4(4)$ \\
Intravenous antibiotherapy (\%) & $54(55)$ \\
\hline
\end{tabular}

SSS, sick sinus syndrome; AVB, atrioventricular block; PICC, peripherally inserted central catheter.

Table 3 . Leadless pacemaker-related complications.

\begin{tabular}{ll}
\hline & ALL (N=99) \\
\hline Implantation failure (\%) & $2(2)$ \\
Complications (\%) Peri-operative ([?]30 days) $(\%)$ & $5(5) 5(5)$ \\
Death* (\%) & $1(1)$ \\
Tamponade (\%) & $3(3)$ \\
Pericardial effusion without drainage (\%) & $1(1)$ \\
Elevated threshold requiring system revision $(\%)$ & $1(1)$ \\
Late (>30 days) (\%) & $0(0)$ \\
\hline
\end{tabular}

* Cardiac tamponade requiring drainage and subsequent multi-system organ failure.

\section{FIGURES}

Figure 1.

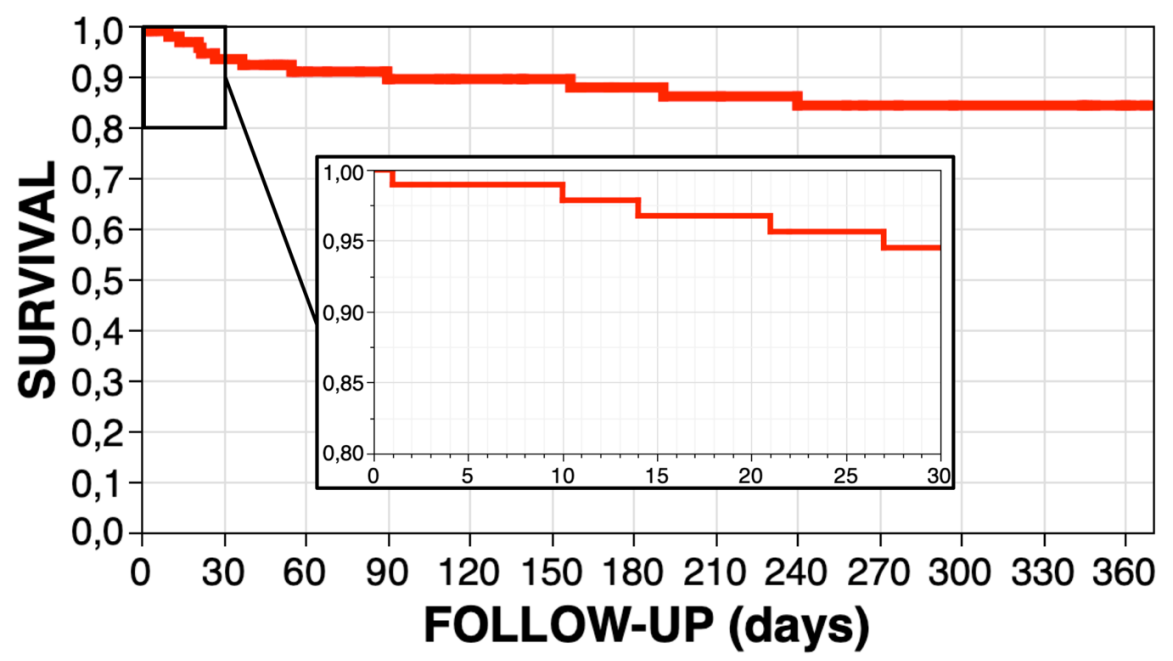

Figure 2. 

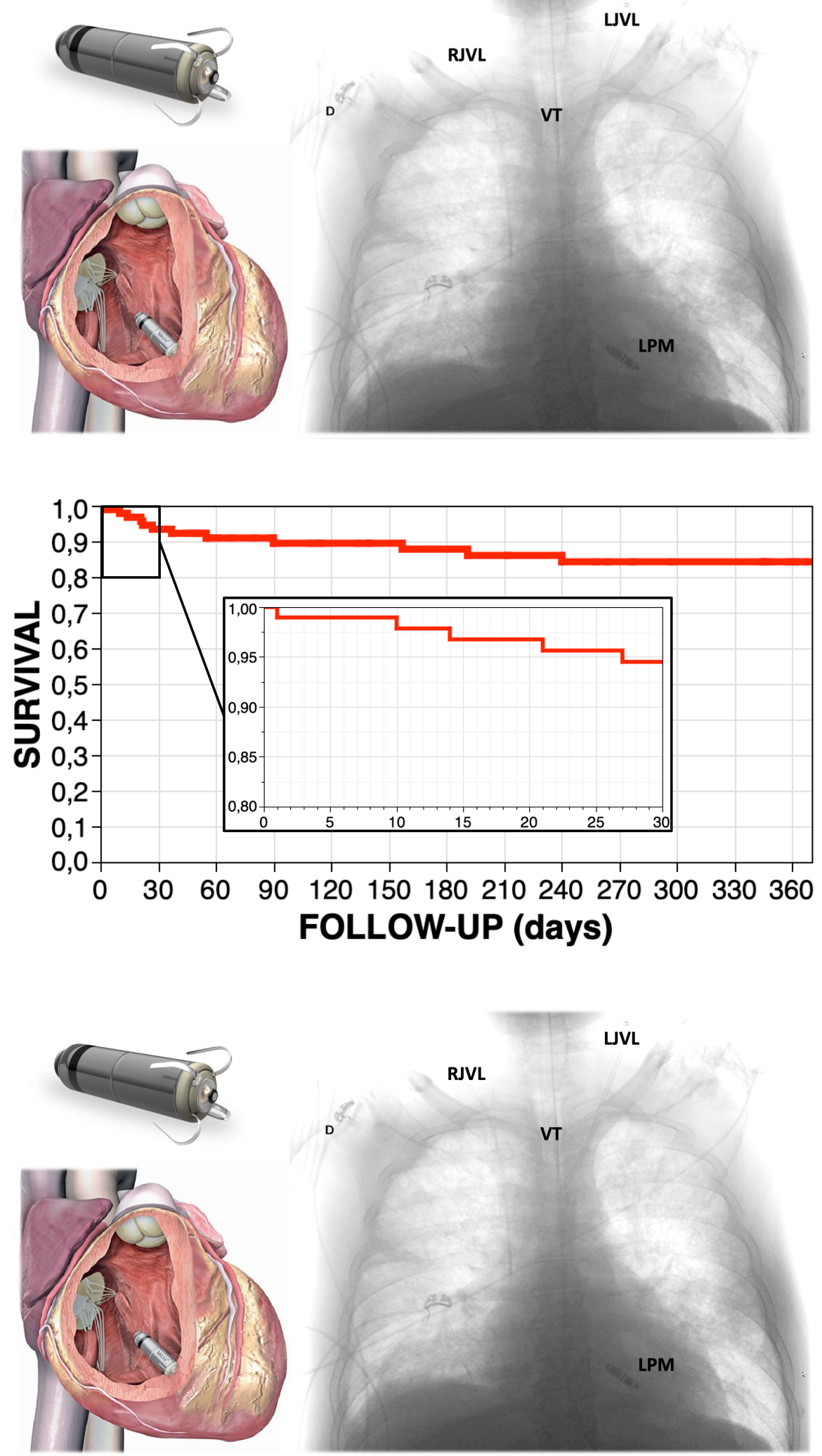


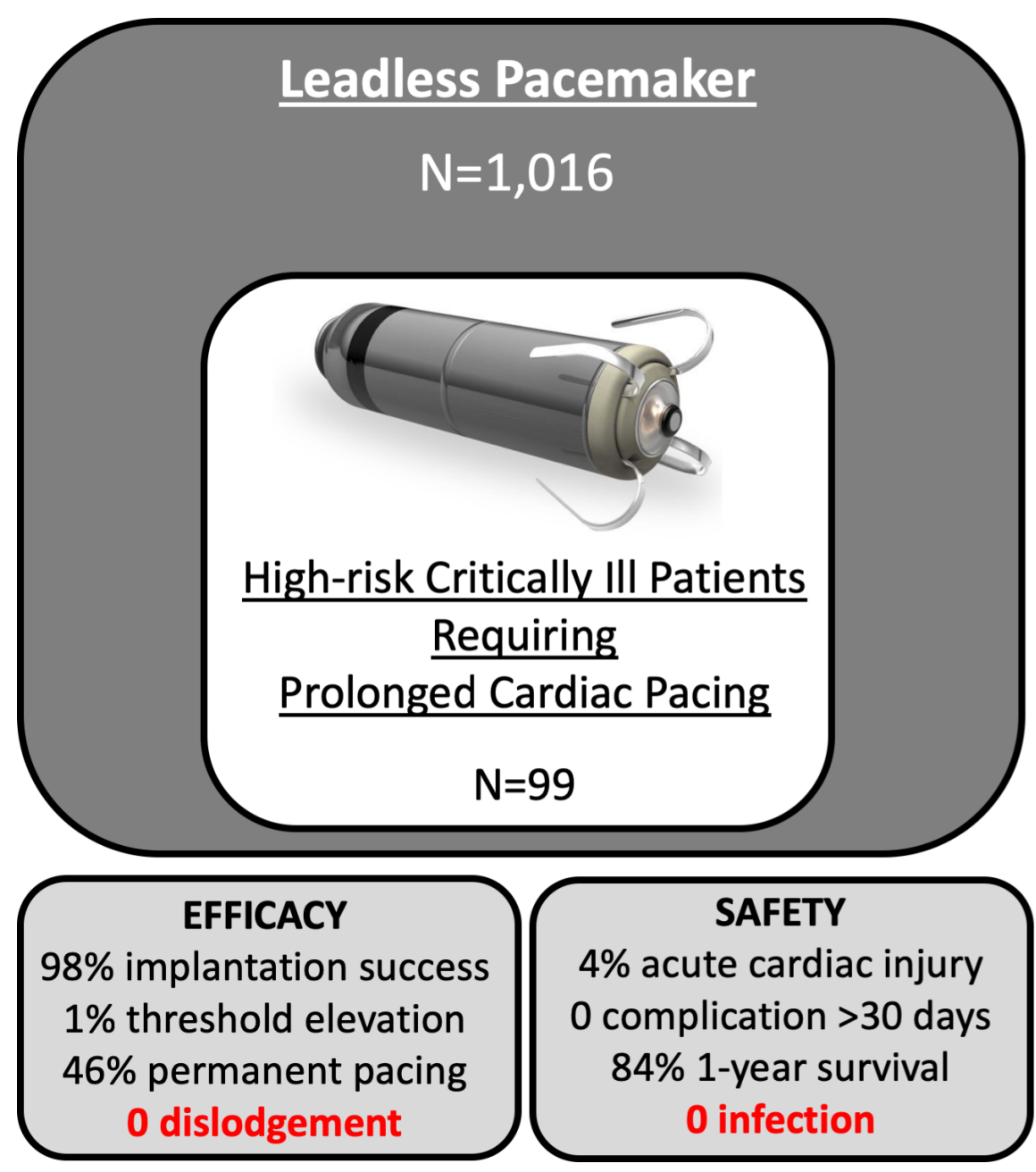

